\title{
New Year, New Name and New Milestones Scope - Journal of Circulating Biomarkers
}

\section{Editorial}

\author{
Shidong Jia ${ }^{1}$ and Winston Patrick Kuo² \\ 1 Genentech Inc, South San Francisco, USA \\ 2 Harvard Medical School, Boston, USA
}

Received 21 Apr 2014; Accepted 29 Apr 2014

DOI: $10.5772 / 58638$

() 2014 The Author(s). Licensee InTech. This is an open access article distributed under the terms of the Creative Commons Attribution License (http://creativecommons.org/licenses/by/3.0), which permits unrestricted use, distribution, and reproduction in any medium, provided the original work is properly cited.

\begin{abstract}
This editorial article introduces a renaming of journal Exosomes and Microvesicles (EXMV) to the Journal of Circulating Biomarkers with a new editorial scope, mission and our approach for the upcoming year in relation to engaging at the international level, the translational art of the study of exosomes and microvesicles, and the interface between exosomes and microvesicles, circulating tumor cells, cell-free circulating DNA and circulating protein markers in precision medicine and drug development. There is a slight change in the members of the Editors in Chief, Editorial Board and extending collaborations to international societies, such as the American Society for Exosomes and Microvesicles (ASEMV).
\end{abstract}

Keywords Exosomes, Microvesicles, Circulating tumor cells, Cell-free circulating DNA, Editorial

In the past year, we have witnessed many excitements, exemplified by our sharp focus on engaging with international key opinion leaders, translational researchers and diagnostic and therapeutic innovators from both academia and industry working in the field of exosomes and microvesicles. We had hoped to leverage EXMV as a niche medium to showcase their latest research and advances, though we only received 13 manuscripts and 10 of which were published requiring the service of 19 individual referees, we have decided to make significant changes to the existing journal. In order to achieve this goal we have decided to implement the following; we have invited a new member to join the EXMV Editors in Chief team, Dr. Shidong Jia (Bio available on the journal website). Dr. Jia is a Scientist at Genentech Inc. where he spearheads the biomarker development efforts in support of Oncology pipeline and drug-companion diagnostic co-development. Dr. Jia is an internationally recognized scientist in the extracellular vesicle field, among other circulating biomarkers and we look forward to working closely with him in bringing international exposure and success to the journal. And when considering the recent breakthrough in blood-based biomarker development, the new team of editors-inchiefs have decided to rename and re-index the journal name from EXMV to "Journal of Circulating Biomarkers" (abbreviated herein as JCB), and to expand the current 
scope from exosomes and microvesicles to the interface between exosomes/microvesicles, circulating tumor cells, cell-free circulating DNA and circulating protein markers in the context of precision medicine and clinical drug development. New discoveries utilizing blood-based biomarkers will potentially enhance our understanding of the pathology and etiology of disease, particularly originating from the genetic and molecular world. The use of exosomes/microvesicles, circulating tumor cells, cell-free circulating DNA and circulating protein markers as a biomarker in clinical medicine for early diagnosis, prognosis and monitoring of therapy will be a significant advancement in the field. Significant progress has been accomplished up to this point, yet we hope this this new renamed journal will be the source to publish the process to the best clinical use of these new discoveries.

As our first task, the Editors in Chief have and will actively expand our Editorial Board and develop collaborations/partnerships with international societies through conferences, meetings, webinars we will be attending, which will include scientific experts who understand how discovery moves from lab to the market and to attract contributors and participation from a more global audience. These experts will serve as the central hub of the circulating biomarker community for the latest advances and provide us guidance.
The JCB manuscript processing and peer-review is entirely online. The Editorial Manager facilitates the manuscript processing time, reducing costs and is a better experience for our authors and reviewers. We have received numerous positive comments on this evolving electronic system, and we are indebted to our publisher, Intech, for managing this program for us. For more information, please visit our new manuscript submission system at http://www.editorialmanager.com/exo/default.asp. In addition, we are enforcing to expedite the acceptance to publishing time for our authors and will request informal opinions from our Editorial Board for borderline cases.

JCB will continue to work on partnering with other scientific societies, academic and industry leaders to develop and advance the circulating biomarker field and to allow free access to knowledge.

It's our pleasure to thank the editorial team and publishers to make this journal possible. 